\title{
Platelet-Derived Microparticle
}

National Cancer Institute

\section{Source}

National Cancer Institute. Platelet-Derived Microparticle. NCI Thesaurus. Code C120026.

A small, membrane bound vesicle circulating in the blood that was shed by a platelet. This is the most abundant type of microparticle in human blood. Since they contain platelet chemokines and surface proteins, these particles may modulate vascular processes, such as blood coagulation. 